REVISTA BRASILEIRA

DE AGROECOLOGIA

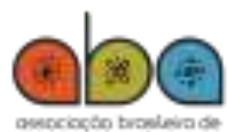

agroecologio

ISSN: 1980-9735

DOI: 10.33240/rba.v14i1.22414

Vol. 14 | No. $1 \mid$ p. 09-13 | 2019

NOTA AGROECOLÓGICA

PREPARADOS HOMEOPÁTICOS NA PRODUÇÃO

DE MELANCIAS

Homeopathic preparations on Watermelon Crop

Mariana Marcolino Costa ${ }^{1}$

1 Docente de Homeopatia no Instituto Tecnológico Hahnemann/Homeobrás Docente no curso de Farmácia da Faculdade Pitágoras, campus Divinópolis Doutoranda em Ciências da Saúde na Universidade Federal de São João delRei, CCO.

E-mails:

marianamarcolinocosta@gmail.com

\section{Recebido em:}

02/08/2017

Aceito para publicação em:

03/12/2018

Correspondência para:

marianamarcolinocosta@gmail.com

\section{RESUMO}

O objetivo do estudo foi relatar o desenvolvimento da cultura de melancias tratadas com preparados homeopáticos. Plantas de melancias foram cultivadas com aplicações de Calcarea carbonica $5 \mathrm{CH}$, Natrum muriaticum $5 \mathrm{CH}$ e Calcarea phosphorica $5 \mathrm{CH}$. Não foi observada a presença de praga ou doença durante o ciclo de plantio e após a colheita. O amadurecimento dos frutos foi adiantado em, aproximadamente, dez dias e o sabor mais adocicado quando comparado a melancias cultivadas com agrotóxicos.

Palavras-chave: Agrohomeopatia. Altas Diluições. Terapias Não Residuais.

\section{ABSTRACT}

The objective of the study was to report the development of the watermelon culture treated with homeopathic preparations. Watermelon plants were cultivated with applications of Calcarea carbonica $5 \mathrm{CH}$, Natrum muriaticum $5 \mathrm{CH}$ and Calcarea phosphorica $5 \mathrm{CH}$. It wasn't observed the presence of any pest of disease during the planting cycle and after harvest. The fruit ripening was advanced in approximately ten days and the sweet flavour accentuated when compared to watermelons cultivated with pesticides.

Keywords: Agrohomeopathy, High Dilutions, Non-Residual Therapies. 
A produção de alimentos, em seu manejo convencional, tem sido questionada de tal modo que práticas adequadas de manejo são uma necessidade (CAVALCANTE et al., 2008). Riscos decorrentes do uso de agrotóxicos têm estimulado a transição para os sistemas de produção orgânica, em que a Homeopatia surge como medida complementar ao manejo sanitário e atende aos pressupostos da produção de alimentos saudáveis e de boa qualidade (JESUS et al., 2018).

O uso da Homeopatia, na agricultura, tem por objetivo proporcionar saúde ao meio rural, com o abandono dos agrotóxicos e fertilizantes, bem como a produção de alimentos que não deixem resíduos químicos no ambiente (MORENO, 2017; ANDRADE e CASALI, 2011). A Homeopatia aplicada à agropecuária é entendida como tecnologia social, em decorrência da baixa dependência por insumos externos e pelo aumento do valor agregado ao produto, propiciando a conservação dos recursos naturais e destacando-se como uma tecnologia limpa e econômica (ANDRADE, 2012; CASALI, 2011).

A homeopatia propicia ao agricultor alcançar o manejo adequado de pragas e doenças, proteção do solo e ambiente, desintoxicação de plantas e redução de gastos financeiros (MORENO, 2017; ANDRADE e CASALI, 2011). O uso de homeopatia na agricultura atua no aumento da imunidade vegetal, sementes mais vigorosas, rendimento de princípios ativos, alteração de padrão energético, adaptação a condições adversas e melhora do estado geral da planta (CAPRA et al., 2014; ANDRADE, 2012). Além disso, apresenta ausência de efeitos colaterais indesejáveis e restabelece a saúde como um todo, considerando as águas, solos, a família do agricultor, a comunidade no entorno da propriedade e o consumidor final do produto (BOFF, 2013).

Sua aplicação na agricultura foi indicada, inicialmente, pela Instrução Normativa no 007, de 17 de maio de 1999, do Ministério da Agricultura, Pecuária e Abastecimento (MAPA), que dispõe sobre normas para a produção de produtos orgânicos vegetais e animais (BRASIL, 1999). Posteriormente, a Lei dos Orgânicos e suas normativas decorrentes, incorporaram a homeopatia como prática sem restrição na produção orgânica, obedecidos os procedimentos previstos em lei. A Instituição Normativa no 17, de 18 de junho de 2014, do MAPA relaciona a homeopatia como uma das substâncias ativas e práticas permitidas para manejo, controle de pragas e doenças nos vegetais, nos tratamentos pós-colheita, assim como para uso como fertilizantes, corretivos e no tratamento a animais de produção (BRASIL, 2014).

Segundo Bonato e Peres (2007), há três modos de se escolher medicamentos homeopáticos para uso na agricultura. O primeiro utiliza de analogia entre os sintomas físicos apresentados na matéria médica para humanos e os vegetais (acognosia). O segundo utiliza preparados ultradiluídos dos agentes causadores de injúria (bioterápicos ou isoterápicos) e o terceiro modo busca usar o próprio elemento faltante como medicamento em soluções ultradiluídas. De acordo com Teixeira e Carneiro (2017), um dos problemas que possa estar restringindo o uso da homeopatia na agricultura é a dificuldade de desenvolver uma matéria médica vegetal, em que sejam compilados sintomas e características das plantas após o uso de medicamentos homeopáticos.

A realização de pesquisas homeopáticas em plantas apresenta inúmeras vantagens à experimentações envolvendo humanos ou animais, pois permite grande número amostral, execução em curto período, baixo custo, inexistência de problemas e ausência de efeito placebo (TEIXEIRA e CARNEIRO, 2017). O objetivo deste estudo foi relatar o desenvolvimento de plantas de melancias tratadas com preparados homeopáticos, além de resposta na produção e qualidade de frutos.

A pesquisa foi realizada na cidade de Carmo do Cajuru, Minas Gerais, em uma área de dois mil metros. Sementes de melancia foram mantidas imersas em recipiente de vidro, contendo água mineral e a homeopatia Calcarea carbonica $5 \mathrm{CH}$ durante 12 horas antes do plantio. Em seguida, foi realizada irrigação única do terreno, prévia ao plantio, com a mesma homeopatia. A irrigação no plantio foi feita uma vez ao dia e a homeopatia era, diariamente, adicionada em uma caixa d'água para essa finalidade. A irrigação foi iniciada no primeiro dia de plantio e durou até que os primeiros frutos estivessem no estádio de vez. Após o plantio das sementes, a utilização da Calcarea carbonica $5 \mathrm{CH}$ foi mantida durante 10 dias. Posteriormente, foi utilizado Natrum muriaticum $5 \mathrm{CH}$ por 10 dias e a terceira aplicação foi com Calcarea phosphorica $5 \mathrm{CH}$, também, por um período de 10 dias. Seguindo essa mesma ordem, os preparados homeopáticos foram alternados, sendo utilizados cada qual por 10 dias, sempre repetindo o ciclo até o final de crescimento das melancias. 
Foram observadas todas as plantas quanto ao tamanho, desenvolvimento e presença de pragas ou doenças. Após dois meses do plantio, as melancias começaram a ser colhidas e todas foram avaliadas quanto ao tamanho, alteração na coloração, presença de rachaduras, de pragas e de doenças. Em relação à parte interna das frutas, foram selecionadas amostras de diferentes pontos da plantação e as mesmas foram analisadas quanto à coloração interna, alterações, qualidade e sabor. Todas essas análises foram realizadas por quatro pessoas.

Comparativamente, o cultivo tradicional com agrotóxicos foi estudado pelos avaliadores que observaram estudos e imagens de plantações e frutos cultivados de forma tradicional. Foram adquiridas melancias cultivadas com o uso de agrotóxicos para serem comparadas às melancias colhidas após o uso exclusivo de homeopatias. Também foram documentados relatos livres de consumidores e proprietários de mercearias e supermercados que adquiriram as melancias.

Em relação às características das plantas observadas, todas se desenvolveram rapidamente $\mathrm{e}$ atingiram altura semelhante ao valor relatado em literatura. Durante todo o desenvolvimento, não foi observada a presença de nenhuma praga ou doença na planta e nos frutos. A ausência de pragas e doenças na plantação, relatada no presente estudo, já foi apresentada na literatura após uso de Calcarea carbonica $4 \mathrm{CH}$ que melhorou a resistência dos frutos a doenças e fortaleceu a planta como um todo (CAPA, 2004). De acordo com Gonçalves e colaboradores, Natrum muriaticum 12CH (GONÇALVES et al., 2011) e Natrum muriaticum 6CH (GONÇALVES et al., 2012) reduziu a incidência de insetos na plantação de cebolas. Todas as homeopatias utilizadas possuem a propriedade de melhorar a utilização de nutrientes pela planta e recuperar plantas desmineralizadas, o que fortalece a planta e frutos frente ao ataque de pragas e doenças (MORENO, 2017; RESENDE, 2014; BONATO, 2006).

As primeiras melancias colhidas adquiriram tamanho e peso rapidamente com, aproximadamente, 70 dias após o plantio, tempo inferior de colheita se comparado aos dados de literatura, em que ela é realizada de 80 a 110 dias após o plantio (BRASIL, 2006). O peso médio foi de dez quilos. Porém, as próximas produções apresentaram o peso reduzido, entre seis e nove quilos, e ao final da colheita os frutos apresentaram peso inferior a cinco quilos. Dados da literatura relatam que cada pé pode produzir até 30 quilos e a produção alcança 20.000 quilos por hectare (BRASIL, 2006).

O número de melancias foi abaixo do cultivo tradicional, provavelmente devido ao espaçamento, densidade de semeadura, desbaste e prolificidade de frutos por planta. A melhoria do peso dos frutos pode ser atribuída ao uso da homeopatia Natrum muriaticum, que é capaz de melhorar a aclimatação e o crescimento da planta e dos frutos (BONATO, 2006; CAPA, 2004). Em outros estudos, o uso de Natrum muriaticum $6 \mathrm{CH}$ aumentou a massa verde e incrementou a taxa de crescimento de feijão (LENSI et al., 2010) e aumentou a taxa de germinação e vigor de sementes de feijão (COPACHESKI et al., 2013). Os benefícios da Calcarea carbonica foram citados em estudo que a utilizou nas potências $6 \mathrm{CH}$ e $12 \mathrm{CH}$ associada à Alumina $6 \mathrm{CH}$ e $12 \mathrm{CH}$, no qual foi observado um aumento do comprimento da raiz e da velocidade de germinação no cultivo de alface (BONFIM et al., 2010).

No presente estudo, foi observada grande quantidade de insetos polinizadores, como as abelhas, na época de floração, o que indica que a homeopatia mantém o equilíbrio ecológico, preservando a fauna e flora benéficas no local. Apenas cinco frutas apresentaram rachaduras e vinte frutas apodreceram antes do amadurecimento. Gonçalves et al. (2015) também encontraram menores tamanhos de peras pós-colheita com associação de Natrum muriaticum $12 \mathrm{CH}$ e calcário de conchas $6 \mathrm{CH}$. O calcário de conchas possui efeito semelhante à Calcarea carbonica e pode atuar no metabolismo de macronutrientes, consumo hídrico e aumento de compostos de defesa em plantas (BONATO, 2006). A área de amarelamento, correspondente à parte em contato com o solo, foi mais extensa quando comparada às melancias cultivadas tradicionalmente com agrotóxicos, mas não interferiu na qualidade do produto.

Ao analisar a parte interna das frutas, foi observado que a coloração vermelha também foi menos intensa quando comparada às cultivadas com agrotóxicos. Não foram observadas alterações no conteúdo interno nas amostras analisadas. Em relação ao sabor, todos os avaliadores relataram um sabor adocicado superior e acentuado quando comparado às frutas cultivadas de forma convencional. Em um estudo com tomates, foi relatado sabor significativamente melhor dos frutos após o uso de medicamentos homeopáticos (NOVOSADYUK, 2011). 
Fator limitação no presente estudo foi o fato de ter sido realizado apenas na primeira plantação feita no terreno, o que já propicia um menor aparecimento de pragas e doenças e dificulta aferir o impacto no uso de homeopatias. Apesar dessas limitações, foi possível observar um ótimo efeito de homeopatias em melancias.

O uso de preparados homeopáticos na agricultura constitui alternativa para a defesa das culturas agrícolas voltadas para a produção de plantas livres de agrotóxicos, a conservação dos recursos naturais, a redução nos custos de produção e aumento na qualidade de vida dos agricultores. Constatouse, com o estudo, proteção na lavoura de melancia às pragas, ao mesmo tempo em que promoveu equilíbrio ecológico. Observou-se redução do tempo de amadurecimento e colheita dos frutos, bem como aumento da qualidade. É fundamental o desenvolvimento de outros estudos, analisando-se diferentes variáveis e dinamizações, frequência e forma de aplicação.

\section{Referências}

ANDRADE, F. M. C. Tecnologia e aplicação da homeopatia na horticultura. Hortic. bras., v. 28, n. 2, 2012.

ANDRADE, F. M. C.; CASALI, V. W. D. Homeopatia, agroecologia e sustentabilidade. Rev. Bras. de Agroecologia. v. 6, n. 1, p. 49-56, 2011.

BOFF, P. Building homeopathy into agro-ecology science. In: II International Conference on Homeopathy in Agriculture. Anais. Maringa, Brazil. p. 1-4, 2013.

BONATO, C. M. Homeopatia simples: Alternativa para a agricultura familiar. Marechal Cândido Rondon: Gráfica Líder, p. 32p, 2006.

BONATO, C. M., PERES, P. G. P. Homeopatia em vegetais. In: VIII Seminário Sobre Ciências Básicas em Homeopatia, 8, 2007, Lages, Anais. Lages: CAV/UDESC; EPAGRI. p.41-59, 2007.

BONFIM, F. P. G.; et al. Germination abnd vigor of lettuce seeds (Lactuca sativa L.) pelleted with homeopathic preparations Alumina and Calcarea carbonica subjected to toxic levels of aluminum. Int J High Dilution Res. v.9, n.33, p.138-146, 2010.

BRASIL. Instrução normativa no 07, de 17 de maio de 1999. Dispõe sobre normas para produção de produtos orgânicos vegetais e animais. Diário Oficial da República Federativa do Brasil, Brasília, v. 99, n. 94, p. 11-14, Seção 1, 1999.

BRASIL. Instrução normativa no 17, de 18 de junho de 2014. Estabelece o Regulamento Técnico para os Sistemas Orgânicos de Produção, bem como as listas de substâncias e práticas permitidas para uso nos Sistemas Orgânicos de Produção. Diário Oficial da República Federativa do Brasil, Brasília, Seção 1, 2014.

BRASIL. Ministério da agricultura. Empresa Brasileira de Pesquisa Agropecuária -EMBRAPA. Como produzir melancia. Brasília, DF: Embrapa Informação Tecnológica. ABC da Agricultura Familiar. v.1, 29 p., 2006.

CAPA - CENTRO DE APOIO AO PEQUENO AGRICULTOR. Homeopatia simples: Alternativa para Pequenos Agricultores. Maringá, PR, 2004.

CAPRA, R. S.; et al. Preparados homeopáticos e ambiente de cultivo na produção e rendimento de quercetina em carqueja [Baccharis trimera (Less) DC.]. Rev. Bras. PI. Med. v.16, n.3, p.566-573, 2014.

CASALI, V. W. D. Utilização da Homeopatia em vegetais. In: SEMINÁRIO BRASILEIRO SOBRE HOMEOPATIA NA AGROPECUÁRIA ORGÂNICA, 5., 2004, Toledo-PR. Resumo dos trabalhos apresentados. Paraná, 2004.

CAVALCANTE, L. F.; et al. dos. Micronutrients and sodium foliar contents of yellow passion plants as a function of biofertilizers. Fruits. v. 63, n.1, p. 27-36, 2008.

COPACHESKI, M.; et al. Revitalização de sementes de feijão (Phaseolus vulgaris) submetidas a tratamentos homeopáticos. Cadernos de Agroecologia, v. 8, n. 2, p. 13538, 2013.

GONÇALVES, P. A. S.; et al. Efeito da aplicação do preparado homeopático Natrum muriaticum na incidência de Thrips tabaci na produtividade e na armazenagem de cebola em sistema orgânico. Agropecuária Catarinense. v. 24, n. 2, 2011.

GONÇALVES, P. A. S.; et al. Dosagens em altas diluições de Natrum muriaticum e calcário de conchas no manejo de tripes, míldio e produtividade de cebola em sistema orgânico. Rev. Bras. de Agroecologia. v.7, n. 3, p. 152160, 2012.

GONÇALVES, P. A. S.; et al. Efeito de altas diluições de calcário de conchas e Natrum muriaticum no manejo fitossanitário, na produtividade e na armazenagem de cebola em sistema orgânico. Agropec. Catarin. v. 27, n. 3, p. 78-82, 2015.

JESUS, R. A.; et al. Influência do medicamento homeopático Sulphur no desenvolvimento de Lactuca sativa L. (Asteraceae). Journal of Agronomic Sciences. v. 7, n. 1, p. 186-193, 2018. 
LENSI, M. M.; et al. A pilot study of the influence of Natrum muriaticum $6 \mathrm{CH}$ and $30 \mathrm{CH}$ in a standardized culture of Phaseolus vulgaris L. Int J of High Dilution Res, v. 9, n. 30, p. 43-50, 2010.

MORENO, M. M. Agrohomeopatía como alternativa a los agroquímicos. Rev Med Homeopat. v. 10, n. 1, p. 9-13, 2017.

NOVOSADYUK, T. V. Plants: a unique model for research on high diluted substances. Int J High Dilution Res. v. 10, n. 36, p. 265-267, 2011.

RESENDE, J. M. (Coordenador). Caderno de Homeopatia - Instruções práticas geradas por agricultores sobre o uso da homeopatia no meio rural, $4^{\mathrm{a}}$ ed. Departamento de Fitotecnia da Universidade Federal de Viçosa. 48 p., 2014.

TEIXEIRA, M. Z.; CARNEIRO, S. M. T. P. G. Efeito de ultradiluições homeopáticas em plantas: revisão da literatura. Revista de Homeopatia. v. 80, n. 1/2, p.113-132, 2017. 